\title{
Comparison of face-to-face interview and telephone interview administration of COPD assessment test: a randomized study
}

\author{
Guilherme F. da Silva $\cdot$ Maria Tereza A. Morano • \\ Maria Penha U. Sales • Natalia B. Olegário • \\ Antonio George M. Cavalcante · Eanes D. B. Pereira
}

Accepted: 17 October 2013

(C) Springer Science+Business Media Dordrecht 2013

\begin{abstract}
Objective To assess the measurement properties of the COPD assessment test (CAT) in a randomized trial comparing a face-to-face interview (FFI) with a telephone interview (TI).

Methods A randomized study was conducted at two teaching hospitals in Fortaleza, Brazil. Patients were randomly assigned to answer the CAT questionnaire either in a FFI or by TI. The two groups were assessed for internal consistency reliability, cross-sectional validity and testretest reliability. All patients performed spirometry and answered the modified medical research council dyspnea scale and the St. George's respiratory questionnaire (SGRQ).

Results The total scores of the CAT questionnaire were similar for face-to-face and TI groups, $20.71(95 \%$ CI 18-23.4) versus 20.81 (95\% CI 19.31-21.7), respectively. For both mode of administration, we found good internal consistency reliability, the Cronbach's alpha ranged from 0.74 (95\% CI 0.61-0.84) to 0.89 (95\% CI 0.84-0.93) for the TI and FFI, respectively. In general, moderate-to-high correlations of CAT with SGRQ were observed, independent of the administration format. For the test-retest reliability, the intraclass correlation coefficients were very similar for both FFI and TI group 0.96 (95 \% CI 0.93-0.97) versus 0.98 (95\% CI 0.96-0.98), respectively.
\end{abstract}

G. F. da Silva · A. G. M. Cavalcante · E. D. B. Pereira ( $₫)$ Universidade Federal do Ceará (UFC), Rua Barbara de Alencar 1401, Fortaleza, Ceará 60140000, Brazil

e-mail: eanes@fortalnet.com.br

M. T. A. Morano · M. P. U. Sales · N. B. Olegário Hospital de Messejana Dr. Carlos Alberto Studart Gomes, Secretaria de Saúde do Estado do Ceará (SESA), Fortaleza, Ceará, Brazil
Conclusion This study demonstrated that the CAT questionnaire administration either in a FFI or by TI presents moderate-to-high measurement properties. This provides support for the use of both modes of questionnaire administration.

Keywords Health status - COPD assessment test (CAT) - Face-to-face interview questionnaire .

Telephone interview questionnaire

\section{Introduction}

Chronic obstructive pulmonary disease (COPD) is one of the leading causes of morbidity and mortality worldwide. It results in a substantial economic and social burden [1,2].

In order to guide therapeutic choices, the assessment of COPD patients must include outcomes that measure the severity of the disease, the impact on patient's health status and the risk of future events (such as exacerbations, hospital admissions and death). The COPD assessment test (CAT) can provide a simple, efficient and reliable tool for assessing and monitoring the impact of COPD regarding the patients' health status [3-5]. The CAT was developed and validated as a self-administered questionnaire [6].

Previous work has revealed that different modes of questionnaire administration may have an influence on its results $[7,8]$. The mode of questionnaire administration has important implications for research methodology, such that the validity of the results and the soundness of public health policies developed from their evidences may be compromised [8].

While the appropriateness of the self-administered CAT in different cultural settings has been demonstrated [6, 9, 10], the self-administrative nature of the questionnaire may 
be a problem for countries with low levels of education. As such, there is a need to assess whether other methods of administration would be important, especially for countries with lower levels of education. While face-to-face interviews (FFIs) are frequently used to administer questionnaires in Brazil [11, 12] and other countries [13], an equivalent telephone administered instrument would be cost-effective, as it would not require that patients to participate at the research centers. However, there is a lack of evidence as to whether the mode of questionnaire administration has an effect on the measurement properties of the CAT [14, 15]. The aim of this study was to assess the psychometric properties of the CAT in a randomized trial comparing the FFI with the TI.

\section{Methods}

Patients and study design

This was a randomized study conducted at two teaching hospitals in Fortaleza, Brazil, from January to December 2012.

The research protocol was approved by local Research Ethics Committee (no. 880/12), and written informed consent was obtained in all cases. Patients were recruited during their initial assessment prior to enrollment in a pulmonary rehabilitation program at one of the two teaching hospitals. To participate in the study, patients had to meet the following criteria: be smokers or ex-smokers with a smoking history of at least 10 pack-years, be between the ages of 40-80 years, have a previous diagnosis of COPD, and have a post-bronchodilator $\mathrm{FEV}_{1}<80 \%$ of predicted and $\mathrm{FEV}_{1} / \mathrm{FVC}<0.7$. Patients with asthma as a primary diagnosis or other active chronic respiratory diseases were excluded. Patients were randomly allocated to complete the CAT as either a FFI or TI. The patients answered the questionnaires twice, 1 week apart. For both methods, the same interviewer used the traditional paper and pencil questionnaire at both time points. The patients were blinded to their previous scores at the follow-up visit and did not undergo any therapy changes.

\section{CAT questionnaire}

The CAT is a trademark of the GlaxoSmithKline group of companies [6]. It has been translated to the Portuguese language by the company. It is available on the website www.catestonline.org.

This questionnaire consists of eight items that describe cough, phlegm, chest tightness, breathlessness going up hills/stairs, activity limitations at home, confidence leaving home, sleep and energy. Each item is scored from 0 to 5 , thereby making the range of the total score from 0 to 40 . Higher scores represent worse health. The CAT questionnaire is designed to assess current health and does not specify a recall period.

Validation instruments to assess cross-sectional validity of CAT

To assess functional capacity, all patients performed spirometry. In addition, we used the dyspnea modified Medical Research Council (mMRC) scale to assess the intensity of perceived dyspnea. This scale is described as follows: 0, breathless with strenuous exercise; 1, slight dyspnea when hurrying on the level or walking up a slight hill; 2, walk slower than people of the same age on the level because of breathlessness; 3, stop for breath after walking about 100 yards or after a few minutes on level ground; 4, too breathless to leave the house or breathless when dressing or undressing.

To assess the patients' health-related quality of life, we used the St. George's respiratory questionnaire (SGRQ). The SGRQ consists of 53 items, which can be aggregated into an overall score and three domain scores describing symptoms, activity and impact. Weighting each item and dividing the summed weights by the maximum possible weight and expressing the result as a percentage provides the patient's score. The scores range from 0 (best) to $100 \%$ (worst). Both the mMRC and SGRQ were performed only by FFIs.

The mMRC and SGRQ are the most widely English language questionnaires used for measuring health status and dyspnea in COPD patients in a research setting. Both were previously translated and validated for the Brazilian Portuguese language [16, 17]. For validation criterion of mMRC, the authors used the SGRQ [16], and for validation of SGRQ, the authors used the functional parameters [17].

Statistical analysis

Analysis focused on the comparison of the face-to-face and TI modes of administration. We used parametric tests to compare the baseline measures of both groups. Thereafter, the psychometric properties for the face-to-face and TI questionnaires were first assessed individually and then compared with each other.

We assessed the internal consistencies for each questionnaire mode by calculating the Cronbach's alpha. In addition for each standardized item, the corrected itemtotal correlation and Cronbach's alpha (excluding the item under study) were calculated.

To assess the cross-sectional validity, we used Pearson correlation coefficients between the total score of CAT, administered by face-to-face and TIs, and the scores of the 
Table 1 Demographic, clinical and functional characteristics of 100 patients, according to face-to-face interview and telephone interview groups

\begin{tabular}{|c|c|c|c|}
\hline Variables & $\begin{array}{l}\text { FFI group } \\
(n=50)\end{array}$ & $\begin{array}{l}\text { TI group } \\
(n=50)\end{array}$ & $p$ value* \\
\hline Age years $($ mean $\pm \mathrm{SD})$ & $66.2 \pm 8.4$ & $67.8 \pm 8.5$ & 0.3 \\
\hline \multicolumn{4}{|l|}{ Gender $^{\mathrm{a}}$} \\
\hline Male $(\%)$ & 48 & 62 & \\
\hline Female $(\%)$ & 52 & 38 & 0.3 \\
\hline $\begin{array}{l}\text { Smoking (pack- } \\
\text { years } \pm \text { SD) }\end{array}$ & $41.4 \pm 11.2$ & $38 \pm 11.1$ & 0.1 \\
\hline \multicolumn{4}{|l|}{ Level of schooling $(\%)^{\mathrm{a}}$} \\
\hline Illiterate & 68 & 64 & \\
\hline Not Illiterate & 32 & 36 & 0.8 \\
\hline $\begin{array}{l}\mathrm{FEV}_{1} \% \text { pred } \\
(\text { mean } \pm \mathrm{SD})\end{array}$ & $44 \pm 13.8$ & $45.8 \pm 13.3$ & 0.5 \\
\hline \multicolumn{4}{|l|}{ SGRQ (mean \pm SD) } \\
\hline Symptoms domain & $41.2 \pm 23.7$ & $46 \pm 22.1$ & 0.3 \\
\hline Activity domain & $55.5 \pm 25.1$ & $66.1 \pm 20.5$ & $0.03 *$ \\
\hline Impacts domain & $37.9 \pm 20.1$ & $43.6 \pm 18.1$ & 0.2 \\
\hline Total score & $44.8 \pm 20.3$ & $51.9 \pm 16.7$ & 0.06 \\
\hline MMRC $($ mean \pm SD) & $1.8 \pm 1$ & $2.2 \pm 0.8$ & 0.3 \\
\hline $\mathrm{CAT}($ mean $\pm \mathrm{SD})$ & $20.7 \pm 9.8$ & $20.8 \pm 7.1$ & 0.9 \\
\hline $\begin{array}{l}\text { Time to answer the CAT } \\
\text { (s) }(\text { mean } \pm \mathrm{SD})\end{array}$ & $104 \pm 0.5$ & $180 \pm 0.4$ & $0.004 *$ \\
\hline
\end{tabular}

FFI face-to-face interview, TI telephone interview, Illiterate having little or no formal education, Not illiterate having formal education, $F E V_{1}$ forced expiratory volume in the first second

* $p$ value from unpaired t-tests comparing FFI with TI

${ }^{a}$ Chi square

Table 2 Mean scores and confidence interval of each individual item and total score of the CAT questionnaire for FFI and TI groups

\begin{tabular}{lccc}
\hline Variables & $\begin{array}{l}\text { CAT-FFI group } \\
(n=50) \\
\text { mean }(95 \% \mathrm{CI})\end{array}$ & $\begin{array}{l}\text { CAT-TI group } \\
(n=50) \\
\text { mean }(95 \% \mathrm{CI})\end{array}$ & $p$ value \\
\hline Cough & $2.51(2.07-2.53)$ & $2.72(2.34-3.10)$ & 0.6 \\
Phlegm & $2.25(1.81-2.69)$ & $2.21(1.83-2.59)$ & 0.9 \\
Chest & $2.11(1.64-2.58)$ & $1.72(1.34-2.11)$ & 0.2 \\
$\quad$ tightness & & & \\
Breathlessness & $3.23(2.82-3.64)$ & $3.54(3.21-3.87)$ & 0.3 \\
Activities & $3.01(2.56-3.44)$ & $3.31(2.98-3.64)$ & 0.1 \\
Confidence & $2.42(1.98-2.86)$ & $2.32(1.91-2.73)$ & 0.7 \\
Sleep & $2.33(1.89-2.77)$ & $2.11(1.64-2.58)$ & 0.5 \\
Energy & $2.91(2.58-3.24)$ & $2.72(2.34-3.10)$ & 0.4 \\
Total score & $20.71(18.01-23.42)$ & $20.81(19.81-21.81)$ & 0.9 \\
\hline
\end{tabular}

FFI face-to-face interview, $T I$ telephone interview, $C I$ confidence interval, CAT COPD assessment test

validation instruments. Finally, we assessed test-retest reliability using intraclass correlation coefficients for the baseline and follow-up scores of both modes of
Table 3 Corrected item-total correlations and internal consistency reliability if item was deleted for scores of FFI and TI modes of CAT questionnaire administration

\begin{tabular}{llllll}
\hline Variables & \multicolumn{2}{l}{ FFI group $(n=50)$} & & \multicolumn{2}{l}{ TI group $(n=50)$} \\
\cline { 2 - 3 } & $\begin{array}{l}\text { Item-total } \\
\text { correlation }\end{array}$ & $\begin{array}{c}\text { Alpha } \\
\text { if item } \\
\text { deleted }\end{array}$ & & $\begin{array}{l}\text { Item-total } \\
\text { correlation }\end{array}$ & $\begin{array}{c}\text { Alpha } \\
\text { if item } \\
\text { deleted }\end{array}$ \\
\hline Cough & 0.74 & 0.87 & 0.44 & 0.72 \\
Phlegm & 0.79 & 0.87 & & 0.45 & 0.71 \\
Chest tightness & 0.69 & 0.88 & & 0.40 & 0.73 \\
Breathlessness & 0.64 & 0.88 & 0.44 & 0.72 \\
Activities & 0.61 & 0.89 & 0.47 & 0.71 \\
Confidence & 0.78 & 0.87 & & 0.49 & 0.71 \\
Sleep & 0.50 & 0.90 & 0.40 & 0.75 \\
Energy & 0.69 & 0.88 & 0.60 & 0.69 \\
\hline
\end{tabular}

FFI face-to-face interview

TI telephone interview

administration. All statistical analyses were performed with SPSS for Windows version 10.0 (SPSS Inc., Chicago, IL).

\section{Results}

A total of 100 patients, 50 for each group, were randomly allocated to participate in the face-to-face or TIs. Patients of face-to-face and TI groups had similar demographic, clinical and functional characteristics, except a higher SGRQ activity domain $(p=0.03)$ and longer time to answer the CAT questionnaire $(p=0.004)$ were observed in the TI group (Table 1).

The scores for each individual item and the total score of the CAT questionnaire were similar for face-to-face and TI groups (Table 2).

\section{Test-retest reliability}

Test-retest reliability coefficients of the 100 patients were similar for both the face-to-face and TIs 0.96 (95\% CI 0.93-0.97) versus 0.98 (95\% CI 0.96-0.98), respectively.

Internal consistency and cross-sectional validity

Cronbach's alpha coefficients were $0.74 \quad$ (95\% CI 0.61-0.84) and 0.89 (95\% CI 0.84-0.93) for telephone and FFIs, respectively. The telephone mode presented lower internal consistency than the face-to-face mode of administration (Table 3).

Corrected item-total correlations tended to be higher for the FFI group (0.50-0.79). As for the TI group, corrected item-total correlation showed the lowest values, but the 
Table 4 Cross-sectional validity of FFI and TI modes of CAT questionnaire administration: correlation of CAT total score with validation instruments

\begin{tabular}{llclc}
\hline Instrument & $\begin{array}{l}\text { CAT-FFI } \\
\text { group } \\
(n=50) \\
\rho\end{array}$ & $p$ value* & $\begin{array}{l}\text { CAT-TI } \\
\text { group } \\
(n=50)\end{array}$ & $p$ value** \\
& -0.25 & 0.07 & -0.19 & 0.6 \\
\hline FEV $_{1}(\%)$ & & & & \\
$\begin{array}{l}\rho G R Q \\
\text { Symptoms }\end{array}$ & 0.60 & $<0.001$ & 0.50 & $<0.001$ \\
$\quad$ domain & & & & \\
$\begin{array}{l}\text { Activity domain } \\
\text { Impacts domain }\end{array}$ & 0.59 & $<0.001$ & 0.40 & 0.003 \\
Total score & 0.51 & $<0.001$ & 0.53 & $<0.001$ \\
mMRC & 0.48 & $<0.001$ & 0.56 & $<0.001$ \\
\hline
\end{tabular}

FFI the face-to-face interview, $T I$ telephone interview, CAT COPD assessment test, $F E V_{1}$, forced expiratory volume, $S G R Q$ Saint George respiratory questionnaire, $m M R C$ modified Medical Research Council

* $p$ value for the Pearson's correlation coefficient between the CATFFI group and $\mathrm{FEV}_{1}(\%)$, SGRQ and mMRC

** $p$ value for the Pearson's correlation coefficient between the CATTI group and $\mathrm{FEV}_{1}(\%)$, SGRQ and $\mathrm{mMRC}$

internal consistency reliability would be markedly improved by analyzing the values of alpha if the item was deleted.

Table 4 addresses the cross-sectional validity of the two modes of questionnaire administration. In general, moderate-to-high correlations of CAT total scores with SGRQ were observed, independent of the format of administration.

For the FFI group, the correlation of CAT total scores with mMRC was significantly higher than for TI group ( $\rho=0.48$ vs $\rho=0.03$; respectively, $p=0.01$ ).

\section{Discussion}

In this paper, we presented the results of a randomized study that compared the administration of face-to-face versus TIs of the CAT questionnaire. Both modes are valid and reliable at assessing the health status of COPD patients.

Questionnaires administration as an interview, either face-to-face or telephone, has a potential for bias: interviewers may ask incomplete or modified questions, and possibly induce the response to one of the alternatives [8, 18-20]. In order to reduce interviewer-related biases, the interviewer was properly trained in the current study. The administration of the TI was not considered to be a problem in our study, since a high percentage of the population in Fortaleza, Brazil, has mobile phones.

In order to avoid any potential for bias in the comparison between the two methods of interview, the patients were randomly assigned to receive either the FFI or the TI of the CAT; comparisons between the two groups were made. This approach has also been used in previous studies on modes of questionnaire administration for the assessment of quality of oral health in children [18] and quality of life in COPD patients [14, 15]. We did not observe any differences between the two groups in any of the eight items or the overall CAT's score.

The test-retest reliability was excellent. The intraclass correlation coefficient values for both face-to-face and TI exceeded our preset threshold of 0.7 .

Correlation with mMRC was higher on the face-to-face compared with the telephone mode of administration. The scale of dyspnea mMRC was applied as a FFI for all respondents. However, the SGRQ was also administered as a FFI and did not show the same difference. One possible explanation for this difference is the lower internal consistency and longer response time for the TIs.

The SGRQ is the most widely used questionnaire for measuring health status in COPD patients in a research setting. Our study showed good correlations of SGRQ scores with both face-to-face and telephone interview CAT total scores. Others authors also showed these correlations; however, self-administered questionnaires were used [6, 21].

Our study showed good internal consistency although the internal consistency of the TI $(0.74)$ was lower than the FFI.

One possible reason for the lower internal consistency of the TIs could be the difficulties placed on the patients to hear and understand the questionnaire over the phone. Both face-to-face and TI modes of administration are reliable with Cronbach's alpha coefficient above 0.70 (0.89 and 0.74 , respectively).

The results of this study are in line with previous studies assessing different questionnaire administration modes to measure health-related quality of life $[14,15,22,23]$.

Strengths and limitations of the study

One important limitation of this study is the small sample size. However, the study was a randomized design and used a well-validated instrument (SGRQ) to assess the cross-sectional validity of CAT in COPD patients.

All study procedures were consistent across the two centers, and the same interviewer was trained to improve adherence to the study protocol.

Another important limitation of this study was the inability to evaluate a self-administered questionnaire group in order to compare with the other two groups (faceto-face and telephone). The sample in this study consisted of elderly and underprivileged individuals; often, these populations report a rate of illiteracy above the mean [16, 24]. In addition, some individuals considered illiterate would be unable to properly respond to a questionnaire. Despite the potential limitations, our CAT score of 20.7 is 
similar to the scores from self-administered CAT applied to patients from USA and European Union showed that the scores ranged from 17.8 to 21.5 [6].

\section{Conclusion}

To our knowledge, this is the first randomized study to compare FFI with TI modes for CAT administration. Our study showed that face-to-face and TIs were efficient mode of questionnaire administration and both had similar measurement properties.

Acknowledgments We thank Dr. McTaggart-Cowan for her generous assistance on language and grammar. The authors wish to thank $\mathrm{MCT} / \mathrm{CNPq}$ for support of this study.

\section{References}

1. The Global Initiative for Chronic Obstructive Lung disease (GOLD). (2008) Executive summary: Global strategy for the diagnosis, management, and prevention of COPD. Medical communications resources, Available from www.goldcopd.com.

2. Lopez, A. D., Shibuya, K., Rao, C., Mathers, C. D., Hansell, A. L., Held, L. S., et al. (2006). Chronic obstructive pulmonary disease: Current burden and future projections. European Respiratory Journal, 27, 397-412.

3. Dodd, J. W., Marns, P. L., Clark, A. L., Ingram, K. A., Fowler, R. P., \& Canavan, J. L. (2012). The COPD assessment test (CAT): Short- and medium-term response to pulmonary rehabilitation. COPD, 9, 390-394.

4. Mackay, A. J., Donaldson, G. C., Patel, A. R., Jones, P. W., Hurst, J. R., \& Wedzicha, J. A. (2012). Usefulness of the chronic obstructive pulmonary disease assessment test to evaluate severity of COPD exacerbations. American Journal of Respiratory and Critical Care Medicine, 1(85), 1218-1224.

5. Ghobadi, H., Ahari, S. S., Kameli, A., \& Lari, S. M. (2012). The relationship between COPD assessment test (CAT) scores and severity of airflow obstruction in stable COPD patients. Tanaffos, $11,22-26$.

6. Jones, P. W., Harding, G., Berry, P., Wiklund, I., Chen, W. H., \& Leidy, N. K. (2009). Development and first validation of the COPD Assessment Test. European Respiratory Journal, 34, 648-654.

7. Tourangeau. R., Rips, L. J., \& Rasinski, K. (2000). The psychology of survey response. In Chapter 10: Mode of data collection. (pp. 289-312) Cambridge: Cambridge University Press.

8. Bowling, A. (2005). Mode of questionnaire administration can have serious effects on data quality. Journal of Public Health, 27, 281-291.

9. Wiklund, I., Berry, P., Lu, K. X., Fang, J., \& Fu, C. (2010). The Chinese translation of COPD assessment test ${ }^{\mathrm{TM}}$ (CAT) provides a valid and reliable measurement of COPD health status in Chinese COPD patients. American Journal of Respiratory and Critical Care Medicine, 181, A3575.

10. Seohyun, L., Lee, J. S., Song, J. W., Chang-Min, C., Shim, T. S., \& Kim, T. B. (2010). Validation of the Korean version of chronic obstructive pulmonary disease assessment test (CAT) and
Dyspnea-12 questionnaire. Tuberculosis and Respiratory Diseases, 69, 171-176.

11. Moreira, G. L., Pitta, F., Ramos, D., Nascimento, C. S. C., Barzon, D., Kovelis, D., et al. (2009). Portuguese-language version of the chronic respiratory questionnaire: A validity and reproducibility study. Brazilian Journal of Pneumology, 35, 737-744.

12. Silva, P. N. C., Jardim, J. R., Souza, G. M. C., Hyland, M. E., \& Nascimento, O. A. (2012). Cultural adaptation and reproducibility of the breathing problems questionnaire for use in patients with COPD in Brazil. Brazilian Journal of Pneumology, 38, 339-345.

13. Pinto, J. M. S., González, J. R., Arenillas, J. I. C., Nogueras, A. M. M., \& Gómez, F. P. (2010). Quality of life related to health of patients with chronic obstructive pulmonary disease and asthma assessed by SGRQ. Portuguese Journal of Pneumology, 16, $543-558$.

14. Schünemann, H. J., Goldstein, R., Mador, M. J., McKim, D., Stahl, E., Puhan, M., et al. (2005). A randomised trial to evaluate the self-administered standardised chronic respiratory questionnaire. European Respiratory Journal, 25, 31-40.

15. Puhan, M., Behnke, M., Frey, M., Grueter, T., Brandli, O., Lichtenschopf, A., et al. (2004). Self-administration and interviewer-administration of the German Chronic Respiratory Questionnaire: instrument development and assessment of validity and reliability in two randomised studies. Health and Quality of Life Outcomes, 2, 1-29.

16. Kovelis, D., Segretti, N. O., Probst, S., Lareau, S. C., Brunetto, A. F., \& Pitta, F. (2008). Validação do Modified Pulmonary Functional Status and Dyspnea Questionnaire e da escala do Medical Research Council para o uso em pacientes com doença pulmonar obstrutiva crônica no Brasil. Brazilian Journal of Pneumology, 35(8), 737-744.

17. Sousa, T. C., Jardim, J. R., \& Jones, P. (2000). Validação do Questionário do Hospital Saint George na Doença Respiratória (SGRQ) em pacientes portadores de doença pulmonar obstrutiva crônica no Brasil. Brazilian Journal of Pneumology, 26(3), $119-125$.

18. Tsakos, G., Bernabé, E., O’Brien, K., Sheiham, A., \& de Oliveira, C. (2008). Comparison of the self-administered and intervieweradministered modes of the child-OIDP. Health and Quality of Life Outcomes, 6, 40-48.

19. Cook, C. (2010). Mode of administration bias. The Journal of Manual \& Manipulative Therapy, 18, 61-63.

20. Delgado-Rodriquez, M., \& Llorca, J. (2004). Bias. Journal of Epidemiology and Community Health, 58, 635-641.

21. Tsiligianni, I. G., der Molen, T., Moraitaki, D., Lopez, I., Kocks, J. W. H., Karagiannis, K., et al. (2012). Assessing health status in COPD. A head-to-head comparison between the COPD assessment test (CAT) and the clinical COPD questionnaire (CCQ). BMC Pulmonary Medicine, 12, 20-29.

22. Cook, D. J., Guyatt, G. H., Juniper, E., Griffith, L., McIlroy, W., Willan, A., et al. (1993). Interviewer versus self-administered questionnaires in developing a disease-specific, health-related quality of life instrument for asthma. Journal of Clinical Epidemiology, 46, 529-534.

23. Weinberger, M., Oddone, E. Z., Samsa, G. P., \& Landsman, P. B. (1996). Are health related quality-of-life measures affected by the mode of administration? Journal of Clinical Epidemiology, 49, 135-140.

24. Education at a Glance 2012: OECD indicators visit: www.oecd. org/edu/eag2012. 